\title{
Goals of surgery and assessment tools for elderly patients referred for cardiac and noncardiac surgery
}

\author{
Flaminia Coccia, Renzo Rozzini \\ Poliambulanza Foundation, Istituto Ospedaliero, Brescia, Italy
}

\begin{abstract}
Surgery in elderly patients is associated with the risk of death, complications, functional decline and disability. Prior to surgery, therefore, an assessment of the health-related priorities, a realistic evaluation of the surgical risks, and individualized optimization of the procedural pathway to follow are mandatory.
\end{abstract}

\section{Introduction}

The risk of periprocedural complications and mortality associated with complex diseases, although it has drastically decreased in the recent past, still remains critically high for patients of advanced age. In elderly patients, as in patients of any age, the greatest risk is of cardiovascular, respiratory and renal complications; added to these, however, there are also specific age-related complications such as malnutrition, cognitive decline, impaired functional status and increased care needs. Cognitive deficit is the principal factor responsible for postoperative delirium [1]. A premorbid functional impairment that is present preoperatively is predictive not only of a worsening of the functional impairment (mild disability can degenerate into dependence) but also of all the other most frequent complications (e.g. infection, non-healing of surgical wounds). Considering that nowadays the indications for surgical procedures are becoming less and less conditioned by age, there is thus an urgent need to understand the potential specific risks linked to advanced age. In the U.S., each year more

Corresponding author: Renzo Rozzini, Direttore Dipartimento Unità di Cura Sub-Intensiva - Unità di Geriatria per Acuti - Unità Sub-Acuti Fondazione Poliambulanza - Istituto 0spedaliero, Via Bissolati 57, 25124, Brescia, Italy. E-mail: renzo.rozzini@poliambulanza.it

Key words: Elderly surgical patient; pre-op evaluation, prognosis.

Received for publication: 21 March 2017

Accepted for publication: 30 March 2017

(C) Copyright F. Coccia and R. Rozzini, 2017

Tipografia PI-ME Editrice, Italy

Monaldi Archives for Chest Disease 2017; 87:849

doi: 10.4081/monaldi.2017.849

This article is distributed under the terms of the Creative Commons Attribution Noncommercial License (by-nc 4.0) which permits any noncommercial use, distribution, and reproduction in any medium, provided the original author(s) and source are credited. than 4 million elderly patients enter the operating room for major surgery. So it is essential to define analytically the aspects of the health status of elderly patients than can influence their perioperative outcomes.

First, it is necessary to define what the goals one hopes to achieve from the surgical procedure are and, at the same time, what the patient's expectations are. In addition, one needs to know what clinical tools to use to evaluate the risk factors for complications.

The first point to establish is what one hopes to obtain from the surgery. The decision or not to operate an elderly patient is based, first of all, on a realistic quantification of the patient's estimated life expectancy and on the risk of perioperative mortality. Even before consulting the surgeon, it is necessary to define the patient's cognitive status, find out what his/her expectations are, the availability of healthcare assistance after the operation, the social context, and the availability of the general practitioner (GP) to actively follow the patient after discharge [2]. The GP, who knows the patient, can provide information about the decision-making capacity of the patient and so facilitate the discussion about the treatment goals. Once the patient's decision-making capacity has been established, it is necessary to clarify what, realistically, the health priorities of the patient are (e.g. to live as long as possible? or to maintain their independence?). Next, one has to evaluate if the potential benefits of surgery (e.g. treating the disease, or alleviating symptoms) are really superior to the risks (e.g. functional decline, death). If surgery is not able to satisfy the goals of the patient, one will have to opt for a noninvasive treatment. Only when patients have been adequately informed and given their consent to surgery can one proceed to evaluate the geriatric conditions that might influence the outcomes of the intervention (e.g. presence of comorbidities, functional dependence, physical performance, mental health state, malnutrition, frailty). At this point, a targeted physical optimization program can (and should) be initiated, involving muscle strength training and nutritional supplements.

\section{Evaluation of the decision-making capacity}

The capacity to make medical decisions includes the capacity to express a choice, the capacity to understand the information related to that choice, and the capacity to understand the consequences of a decision. Aging is per se associated with a loss of cognitive functions and decision-making capacity. Even if it is common to find an impaired decision-making capacity among very elderly patients, age is not the only risk factor for neurocognitive disturbances such as dementia or delirium. A review that evaluated the decision-making capacity of elderly patients in relation to therapeutic choices showed that only $2.8 \%$ of the 'oldest-old' age-group in good health have no decision-making capacity as opposed to $20 \%$ of people with mild cognitive decline and $54 \%$ of people with Alzheimer's disease (patients with moderate or severe dementia are unable to make decisions related to their medical care 
needs); patients with a lesser degree of impairment are able to make only limited decisions while patients with mild cognitive deficit or with minor psychiatric diseases may be able to make simple decisions but certainly not to make complex decisions.

\section{Defining the treatment}

For elderly or very elderly patients with multiple conditions for whom a surgical intervention is theoretically indicated, the goal-oriented approach is preferable to the more traditional disease-oriented approach. The physician needs to help the patient define and formulate precisely their personal expectations. In evaluating a patient, the very first step should be to establish the general life goals of the patient. Following this, one can proceed to describe the disease prognosis and its treatment, and finally sum up the situation with recommendations.

The questions to put to the patient must be easy to understand. For example, to determine the principal aim of a treatment, the doctor could ask "Which of the following goals is most important in this phase of your life: to live for as long as possible? to live independently?". It is also important to help patients express any undeclared preoccupations they might have through the use of clear, unambiguous questions such as "Is there anything in this phase of your life that has been really preoccupying you?". All members of the healthcare team can contribute to clarifying the goals and priorities of the patient. The priorities once articulated can, however, change and they need to be reformulated (i.e. the patient needs to be requested once again) each time a change is detected in the patient's health status (Table 1).

Once the priority of the goals has been established, the surgeon should discuss the feasibility of the intervention. Some treatments are complex and burdensome. If the social support is not adequate, this may preclude the possibility of a complex treatment. The prognosis must influence the choice of treatment. For example, the decision to operate an asymptomatic tumor in the early phase is different in a strong elderly patient compared to one who is frail, or who has a limited life expectancy. Even if age is important in determining the chances of survival, multimorbidity and functional impairment can strongly influence the overall prognosis.
Today several instruments for evaluating the individualized perioperative risk are available. These tools are not specific for the elderly patient and no single instrument incorporates specific geriatric parameters. They are nevertheless indispensable as a starting point for discussion about the surgical risk. The American College of Surgeons National Surgery Quality Improvement Program has developed and validated a surgical risk calculator which estimates the chances of an unfavorable outcome (e.g. complication or death) in patients candidate for surgical treatment (http://riskcalculator.facs.org). It includes some geriatric parameters such as age, functional health status, and albumin level, but some risk factors which are fundamental for predicting adverse events in the very elderly are not included. No information is available on the survival and on the quality of life of elderly patients with surgical conditions who have chosen not to undergo surgery.

\section{Preoperative assessment and optimization of pre-surgical condition in very old patients}

When it has been established that surgery has more probability of yielding benefits than risks, the next step is to identify the modifiable risk factors that might condition the outcomes of a surgical intervention [3]. This assessment must include specific geriatric domains. In theory, specific evaluations are mandatory for all patients in the oldestold age-group who have an indication for surgery, bearing in mind that older patients have a substantially greater risk of morbidity and mortality with respect to younger patients, even for the less complex procedures (e.g. cholecystectomy).

\section{Which assessment tools?}

Specific tools to investigate the health status of the frail person have long existed, and examine the various functional aspects which contribute to determine frailty. These tools are designated by the umbrella term 'multidimensional assessment'. The focus of the multidimensional assessment is on the risk factors for a decline in health (prevalently functional loss) and on the outcomes of specific interventions aimed to

Table 1 . The elderly surgical patients: goals, benefits and risks.

\begin{tabular}{|c|c|}
\hline The elderly surgical patient & Goals of surgery (to discuss with the patient and their family) \\
\hline $\begin{array}{l}\text { - Cardiovascular, respiratory and renal diseases are the most frequent } \\
\text { problems and the ones that contribute most to perioperative } \\
\text { complications in elderly surgical patients } \\
\text { - Alongside these comorbidities, which are shared in common with } \\
\text { young adults, elderly patients can also have a condition of "malnutrition", } \\
\text { and cognitive and functional impairments } \\
\text { - Cognitive impairment predisposes to postoperative delirium } \\
\text { - Premorbid functional impairment limits the patient's mobility; } \\
\text { functional impairment is an independent risk factor for perioperative } \\
\text { complications and for surgery-related functional decline }\end{array}$ & $\begin{array}{l}\text { - A longer life } \\
\text { - Improved functional status and independence } \\
\text { - Maintaining integrity of one's mental status } \\
\text { - Comfor }\end{array}$ \\
\hline \multicolumn{2}{|c|}{ Evaluate surgery's potential to satisfy the patient's goals and priorities } \\
\hline Potential benefits & Potential risks \\
\hline $\begin{array}{l}\text { - Treatment of the disease } \\
\text { - Longer life } \\
\text { - Treatment-control of symptoms } \\
\text { - Improvement of functional status (i.e. recovery of joint mobility) }\end{array}$ & $\begin{array}{l}\text { - Premature death } \\
\text { - Delirium, worsened cognitive status } \\
\text { - Worsening of functional status } \\
\text { - Loss of independence (i.e. } \rightarrow \mathrm{NH} \text { ) }\end{array}$ \\
\hline
\end{tabular}

NH: Nursing Home. Adapted from: Laurence B et al, JAMA 2014. 
combat this negative trend [4-6]. Considering the high number of negative and positive factors (i.e. that exert a protective role towards an adverse event) identifiable in advanced age, the question of what to evaluate is without doubt very difficult. The preoperative geriatric assessment is based on the multidimensional assessment and considers those aspects that play a role in determining independently the major postsurgical outcomes and in modifying the health trend after a procedure (Table 2).

\section{Evaluation of somatic disorders}

In the context of the pre-surgical somatic assessment, problems related to the organs of sense, deconditioning, incontinence and nutritional status play a special role [7]. Nutritional status requires particular attention in that it has been identified as one of the most sensitive markers of negative outcome; an inadequate nutritional status is a biological signal not so much (and not only) of insufficient food intake but rather of a poor homeostatic reserve. This also explains its role as an independent risk factor of post-procedural mortality [8].

Also the determination of co-existing biomedical conditions (i.e. polypathology, multimorbidity, comorbidities) is crucial for defining the prognosis as well as for the therapeutic choices to be made [9]. The assessment tools available estimate the impact of these co-existent conditions on the biological systems in terms either of their cumulative effect - e.g. the Charlson Comorbidity Index [10] and Cumulative Illness Rating ScaleCIRS [11] - or of their interactive effect - e.g. the Geriatric Index of Comorbidity [12]. The definition of multimorbidity-comorbidity represents an advance in the understanding of the somatic health of the patient: when placed in relation with the eventual co-existence of other diseases and their burden of disease, it enables to define with more accuracy the therapeutic spaces, the impact on disability, and the patient's prognosis.

Elderly people with a high index of comorbidity have an increased risk of malnutrition [13]. A low body mass index or overweight are often indicative of a loss of muscle mass, of a possible impairment of the immune system, of increased complications (consequent to an intervention or a disease) and mortality. The Mini Nutritional Assessment (MNA) is the most widely used assessment tool to identify elderly people who are at risk of malnutrition. The MNA has been validated in numerous clinical studies and in different care settings and showed an acceptable reproducibility [14].

\section{Evaluation of mental status}

The psychological evaluation of the elderly patient takes into account diverse aspects of their cognitive, affective and behavioral functions. The assessment tool most used for quantifying cognitive deficit is the Mini Mental State Examination-MMSE [15]. In the setting of psychological stress, depression occupies a central role due to its high prevalence. In the elderly, the manifestations connected to a reduced mood tone can be "masked" (e.g. as sleep disturbances, loss of appetite, constipation), and may appear similar to those commonly found in normal aging or in situations of brain damage. Their assessment provides an indication about the patient's motivation to undergo a demanding procedure. A useful and easy instrument for identifying depressive symptoms is the Geriatric Depression Scale-GDS [16].

\section{Assessment of functional status}

Functional assessment (i.e. assessment of disability) is the principal aspect of geriatric assessment. It enquires about the capacity to perform activities, from the simplest to the most complex, and to maintain a social role. In relation to their complexity and difficulty, the activities of daily living can be defined as "basic" (Basic Activities of Daily Living-BADL), "instrumental" (Instrumental Activities of Daily LivingIADL) or "advanced" (Advanced Activities of Daily Living-AADL) [17]. An impairment of functional status, in all its manifestations, represents a risk for complications, mortality, and increased need for care and assistance [18].

BADLs include the basic functions connected to the capacity to care for one's own self, e.g. walking, getting dressed/undressed, eating, personal hygiene and sphincter control. The most widely used scales that investigate BADLs are the Index of Independence in Activities of Daily Living of Katz and the Barthel Index. The IADLs include complex functions like shopping, managing money, cooking, household maintenance, and using the telephone. Independence in the IADLs is of extreme importance in that it more or less indicates whether a person is able to live independently. One example of a scale which investigates the IADLs is that of Lawton and Brody [19]. The measurement of BADLs, which refer chiefly to actions concerning care of one's own self is, at least in part, independent of environmental and

Table 2. The elderly surgical patients: the prediction of geriatric outcomes.

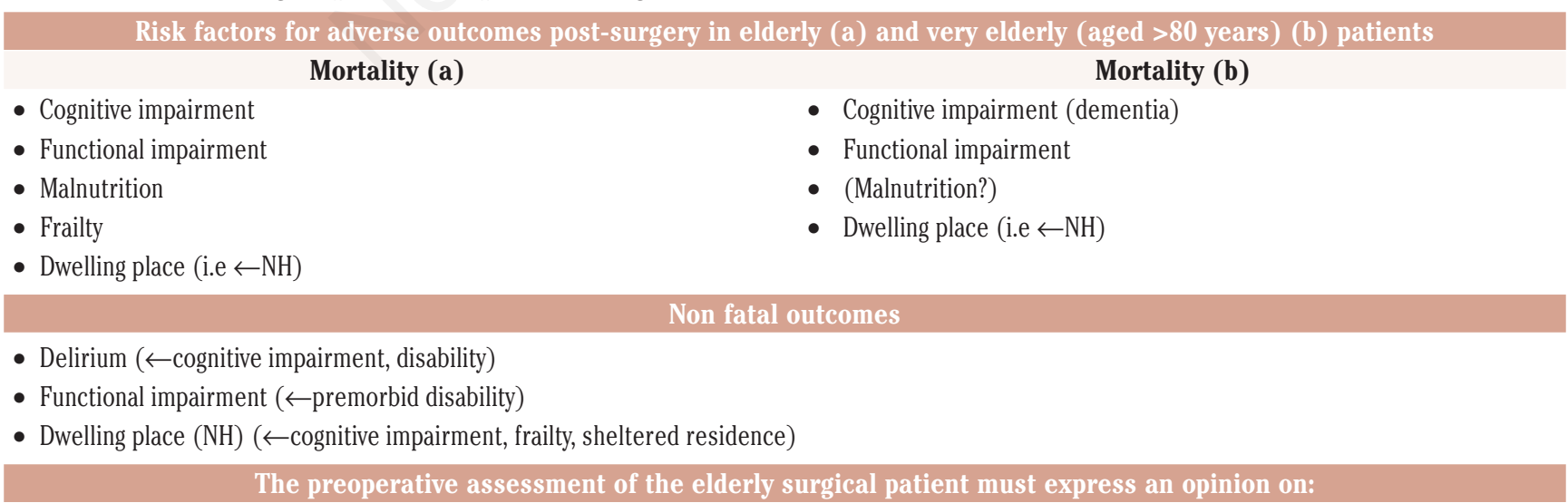

- Risk of premature death (consider specific geriatric items)

- Delirium, worsening of cognitive status

- Worsened functional status

- Loss of independence (i.e. $\rightarrow \mathrm{PAC} \rightarrow \mathrm{NH}$ )

PAC: Post Acute Care; NH: Nursing Home. Adapted from: Laurence B et al, JAMA 2014. 
cultural factors. On the contrary, the capacity to perform the activities considered among the IADLs can be substantially influenced by factors such as the cultural milieu, gender, living habits, social organization, and behavioral problems linked to family dynamics, and thus it may only partially be an indicator of the health status and of the level of physical functioning.

The tools that measure BADLs and IADLs are poorly sensitive as measures for identifying functional deficits in most people in the elderly age category: with these instruments, it is possible to identify only subjects with severe disability (approximately 10\% of elderly people living at home with the BADLs, and 50\% with the IADLs).

A possible alternative to, or integration of, the assessment of BADLs and IADLs, which by definition are subjective (i.e. self-reported or reported by family members), are the objective measures or "performance" tests - e.g. Tinetti Balance and Gait Assessment [20], Physical Performance Test-PPT [21], Timed Up and Go Test [22], and the Short Physical Performance Battery-SPPB [23]. These scales consist of asking the subject to perform a precise task and measuring, according to pre-set criteria, certain parameters (e.g. how many times the subject manages to perform an action, or how long it takes). Objective tests, especially when they contain quantitative assessments (e.g. the time taken to complete a task or the score obtained), show good sensitivity also in detecting minimal changes; they are thus ideal as tests used to integrate the clinical assessment by measuring the effects of a surgical treatment.

\section{Evaluation of social status}

In the geriatric multidimensional assessment, it is also indispensable to analyze the social support network and the economic conditions of the patient: the social support is of great importance in the health of the elderly person [24]. The possibility of living in one's own home, even with a high level of functional impairment, depends on the availability of adequate caregiver support which, in the absence of an institutional or formal type of support, is usually provided by the wife or by adult children (this support is defined as "informal"). In practice, the patient is considered as having an adequate social network if the wife/husband or adult children are present in the same household or if, in the case of disability, one of these family members takes part in the caring. Hence, quantitative parameters provide only an estimate of the theoretic potential of the network of informal support; their information is inadequate if it is not completed with variables of a qualitative type, i.e. the level of social and emotional accompaniment, the degree of intimacy and sharing, personal availability, etc.

\section{Who makes the assessment?}

In general, the assessment of a patient can be done by diverse professional figures. In general, the more structured the assessment, the lower the level of specialization required. It is important to conjugate the assessment tool with the assessor: in some cases, the skill of an assessor can interfere with the accuracy of the assessment (e.g. if the assessor makes evaluative inferences). When the aim of the assessment is to gather clinical information, it can be performed as a preliminary screening tool and then any problems identified can be further investigated through more detailed evaluative instruments [25]. These can (or must) be administered by specialized personnel. In the case of the pre-surgical evaluation, the geriatrist is the professional figure with the most useful expertise for incorporating and integrating basic skills with specialist skills in a pre- and post-procedural care plan $[17,26]$.

\section{Conclusions}

The health status of an elderly person influences the surgical outcomes. Before any intervention, it is necessary to define what the goals are, as well as the patient's expectations and priorities. One must also consider whether the patient's estimated life expectancy justifies the indication for surgery and, if not, whether one should opt for alternative solutions [27]. Very elderly patients with a theoretic surgical indication should undergo an assessment which includes the evaluation of comorbidities and of their geriatric conditions. The personalized preoperative strategies of optimization must focus not only on the biomedical outcomes, but also on the functional, cognitive, nutritional and social outcomes.

When one is consulted about the indications for a surgical intervention in an elderly patient (in order to obtain an informed consent), it is necessary that the patient know what the goals of the treatment are and understand the realistic probabilities of the surgical risk. A preoperative geriatric multidimensional assessment is the most advanced mode for ensuring that this communication is carried out in the most realistic way.

\section{References}

1. Tomlinson JH, Partridge JS: preoperative discussion with patients about delirium risk: are we doing enough? Perioper Med (Lond) 2016;5:22.

2. Ronning B, Wyller TB, Nesbakken A, et al. Quality of life in older and frail patients after surgery for colorectal cancer- a follow-up study. J Geriatr Oncol 2016;7:195-200.

3. Lee YH, Oh HK, Kim DW et al.: use of a comprehensive geriatric assessment to predict short-term postoperative outcome in elderly patients with colorectal cancer. Ann Coloproctol 2016;32:161-9.

4. McDonald SR, Heflin MT: update on preoperative assessment for geriatric patients prior to elective surgery. Geriatr Nurs 2016;37: 160-2.

5. Pilotto A, Cella A, Pilotto A, et al. three decades of comprehensive geriatric assessment: evidence coming from different healthcare settings and specific clinical conditions. J Am Med Dir Assoc 2017;18:192.e1-192.e11.

6. Huisman MG, Kok M, De Bock GH, et al. Delivering tailored surgery to older cancer patients: preoperative geriatric assessment domains and screening tools - a systematic review of systematic reviews. Eur J Surg Oncol 2017;43:1-14.

7. Dupuis M, Kuczewski E, Villeneuve L et al. Age nutrition chirurgic (ACN) study: impact of a geriatric intervention on the screening and management of undernutrition in elderly patients operated on for colon cancer, a stepped wedge controlled trial. BMC Geriatr 2017:7:10.

8. Mazzola P, Ward L, Zazzetta S et al.: association between preoperative malnutrition and postoperative delirium after hip fracture surgery in older adults. J AM Geriatr Soc. 2017 Mar 6.

9. Rozzini R, Bianchetti A, Franzoni F, et al. Social, functional and health status influences on mortality: consideration of a multidimensional inquiry in a large elderly population. J Cross-Cult Gerontol 1991 6:83-90.

10. Charlson ME, Pompei P, Ales KL, et al. A new method of classifying prognostic comorbidity in longitudinal studies: Development and validation. J Chronic Dis 1987;40:373-83.

11. Parmalee PA, Thuras PD, Katz IR, Lawton MP. Validation of the cumulative illness rating scale in a geriatric residential population. $\mathrm{J}$ Am Geriatr Soc 1995;43:130-7. 
12. Rozzini R, Frisoni GB, Ferrucci L, et al. Geriatric index of comorbidity: Validation and comparison with other measures of comorbidity. Age Ageing 2002;31:277-85.

13. Geurden B, Franck E, Weyler J et al. The risk of malnutrition in community-living elderly on admission to hospital for major surgery. Acta Chir Belg 2015;115:341-7.

14. Rubenstein LZ, Harker JO, Salva A, et al. Screening for undernutrition in geriatric practice: developing the short-form mini nutritional assessment (MNA-SF). J Geront 2001;56A:M366-77.

15. Folstein MF, Folstein SE, McHugh PR. Mini-mental state: A practical method for grading the cognitive state of patients for the clinician. J Psychiatr Res 1975;12:189-98.

16. Yesavage JA, Rose TL, Lum 0, et al. Development and validation of geriatric depression screening: a preliminary report. J Psychiatr Res 1983;17:37-49.

17. Kane RA, Kane RL. Assessing the elderly: A practical guide to measurement. Lexington, Massachussets, D.C. Health, 1981.

18. Rozzini R, Frisoni GB. Bianchetti A, et al. Physical performance test and activities of daily living scales in the assessment of health status in elderly people. J Am Geriatr Soc 1993;41:1109-113.
19. Lawton MP, Brody EM. Assessment of older people: self-maintaining and instrumental activities of daily living. Gerontologist 1969;9:179-86.

20. Tinetti ME. Clinical practice: preventing falls in elderly persons. $\mathrm{N}$ Engl J Med 1986;348:42-9.

21. Reuben DB. Sin AL. An objective measure of physical function of elderly outpatients. J Am Geriatr Soc 1990;38:1105-12.

22. Podsiadlo D, Richardson S. The time "Up \& Go": A test of basic functional mobility for frail elderly persons. J Am Geriatr Soc 1991;39:42-8.

23. Guralnik JM, Branch LG, Cummings SR, Crib JD. Physical performance measures in aging research. J Gerontol 1989;44:141-6.

24. Rubenstein LZ, Wieland D, Bernabei R. Geriatric assessment technology: the state of art. Milano: Editrice Kurtis, 1995.

25. Knittel JG, Wildes TS: preoperative assessment of geriatric patients. Anesthesiol Clin 2016;34:171-83.

26. Kane RL, Kane RA, eds. Assessing older persons: Measures, meaning, and practical application. New York, NY: Oxford University Press; 2000.

27. Oresanya LB, Lyons WL, Finlayson E. Preoperative assessment of the older patient. A narrative review. JAMA 2014;311:2110-20. 